\title{
Ankle Joint Pressure Change Before and After Subtalar Joint Arthrodesis in Varus and Valgus Malalignment of the Tibia
}

\author{
$\mathrm{Mu} \mathrm{Hu}$ \\ Ruijin Hospital, Shanghai Jiaotong University School of Medicine \\ Xiangyang $\mathrm{Xu}$ \\ Ruijin Hospital, Shanghai Jiaotong University School of Medicine \\ Jiong Mei ( $\square$ ningquejy@163.com ) \\ Tongji University
}

\section{Research Article}

Keywords: Ankle joint, subtalar arthrodesis, joint pressure, varus ankle

Posted Date: October 25th, 2021

DOI: https://doi.org/10.21203/rs.3.rs-965451/v1

License: (c) (i) This work is licensed under a Creative Commons Attribution 4.0 International License.

Read Full License 


\section{Abstract}

Background: The compensation mechanism of subtalar joint in ankle with varus or valgus deformity is controversial and not well established. This biomechanical study aims to investigate how subtalar joint arthrodesis will affect the ankle joint pressure in varus and valgus malalignment of the tibia.

Methods: 8 fresh-frozen human cadaver legs were tested in this study. A custom-made fixture was utilized and a total of $600 \mathrm{~N}$ was applied to simulate weight-bearing. Intra-articular sensors (TeckScan) were inserted in the ankle joint to demonstrate the ankle joint pressure. conditions include: neutral, $5^{\circ}$, $10^{\circ}, 15^{\circ}$ and $20^{\circ}$ varus, $5^{\circ}, 10^{\circ}, 15^{\circ}$ and $20^{\circ}$ valgus.

Results: After the fusion of the subtalar joint, when the tibia is gradually inverted, the inside pressure of the ankle joint gradually increases, and the pressure on the outside of the ankle joint gradually decreases. When the tibia is gradually eversion, the pressure on the outside of the ankle joint gradually increases, and the inside of the ankle joint gradually decreases.

Conclusions: After the subtalar joint is fused, the compensatory activity of the subtalar joint disappears, and the regulation of the pressure in the ankle joint will be lost.

\section{Background}

Ankle arthritis is a common disease affecting approximately $1 \%$ adult population worldwide[1, 2]. The most common cause of ankle OA is trauma [3,4]. Ankle arthritis is usually accompanied by varus or valgus type deformity. The varus or valgus usually involve the ankle or subtalar joint [5]. The biomechanical relationship between ankle joint and subtalar joint is complex. Whether the subtalar joint will compensate the deformity of ankle joint is controversial $[6,7]$.

A previous study found a lateral shift of the COF and lateral stress concentration for slight varus tibia. However, for more severe varus tibia, the COF shifted medially and decreased the lateral stress concentration[8]. This may explain the compensation relationship between ankle and subtalar joints.

The subtalar joint is an important joint of the hindfoot, which plays a vital role in the ankle varus deformity and the adaptation of the foot to uneven roads during walking. It is hard for ankle arthritis with varus or valgus deformity to manage the balance between supramalleolar and hindfoot using either supramalleolar osteotomy or total ankle replacement. The same issue exists in foot surgeries affecting ankle joints.

Ankle fusion, subtalar fusion or triple arthrodesis are common procedures for ankle or subtalar deformities or arthritis [9-12]. However, after subtalar joint fusion, will it have a corresponding impact on the biomechanics of the ankle joint? This study uses a biomechanical approach to study the influence of subtalar joint fusion on the pressure distribution in the ankle joint. A previous study reported that, for varus ankle, the pressure on the lateral aspect of the ankle joint would increase as the result of subtalar 
valgus inclination. When the varus deformity progressed, the lateral pressure decreased [8]. We hypothesized that this phenomenon would not happen when the subtalar joint is fused.

\section{Methods}

A total of 8 fresh-frozen human cadaver legs were subject to biomechanical testing, the specimens were thawed to room temperature $\left(24^{\circ} \mathrm{C}\right)$. The mean age of the cadavers was $71.4(7.4)$ years old. X-rays were obtained for each specimen and none of them had malalignment of the tibia, hindfoot, nor preexisting subtalar joint osteoarthritis. All specimens had a normal motion of both ankle and subtalar joints. The specimens were tested under two circumstances, the subtalar joint was intact and fused. We tested the change in ankle pressure with tibia varus and valgus at different angles.

The anterior soft tissue (including skin, subcutaneous tissue, anterior joint capsule, tendons and neurovascular bundles) of the ankle joint were dissected to access the ankle joint. Both the medial and lateral ankle ligaments were well preserved. The tibia and fibula were cut at $20 \mathrm{~cm}$ above the ankle joint. For each specimen, the proximal tibia and fibula were potted securely into a custom-made shell, and then mounted on a custom-made fixture. The tibia and fibula were embedded and securely fixed into the shell using dental gypsum. The load was applied to the tibia and fibula via the custom-made shell. Each specimen must be potted in a neutral position, no plantarflexion or dorsiflexion of the ankle joint in the sagittal plane, and no varus or valgus malalignment of the hindfoot in the coronal and no internal or external rotation of the foot in a horizontal plane.

The custom-designed fixture was subjected to testing. Spirit levels were utilized to make sure both the working table and the top plate were horizontal throughout the testing process.

The malalignment of tibia $\left(0^{\circ}, 5^{\circ}, 10^{\circ}, 15^{\circ}, 20^{\circ}\right.$ of tibial varus and valgus) was simulated using a custommade apparatus. Each hole on the apparatus represented a specific angle. A bolt was used to fix the specimen at a desired angle.

The four threaded polyethylene pillars were used to connect the top plate, the compressive forces were exerted via the four pillars. Sensor cells were placed in each pillar, a monitor was connected to each sensor cell, displaying the real-time force. Springs were placed right above each force sensor, then followed by nuts, compressive forces could be generated by twisting the nut on the spring.

The sensor pads (Model 6900, TekScan, Inc., South Boston, MA), with each pad measuring $14 * 14 \mathrm{~mm}$, each pad had 121 senels ( $11 * 11$ sensels), the column and row spacing were $1.3 \mathrm{~mm}$, resulting in a spatial resolution of $0.62 \mathrm{~mm}^{2}$ per sensel. Two pads were put side by side within the ankle joint to measure the ankle joint pressure. The sensor pads were inserted into the ankle joint from anterior and secured by thumbtacks to the distal tibial metaphysis and the foot to avoid sensor motion during testing. The sensor pads were connected to the handle that could be further connected to a personal computer, data including pressure, a force was collected using I-Scan software. 


\section{Situation A: when the subtalar joint was intact}

The baseline ankle joint pressure distribution was initially collected for each specimen. The specimen was fixed at $0^{\circ}$ of tibial varus by inserting a bolt, and the foot was then placed onto the floor freely. A compressive force was generated through the 4 pillars by twisting the nuts. Make sure that the top plate is maintained horizontally throughout the testing. A $600 \mathrm{~N}$ compressive force was applied to simulate the normal load within the ankle joint during ambulation. Both the medial and lateral ankle joint pressure data were collected. Then, the tibial was fixed to $5^{\circ}, 10^{\circ}, 15^{\circ}, 20^{\circ}$ valgus and varus, and the corresponding pressure within the ankle joint was measure and recorded.

\section{Situation B: When the subtalar joint was fused}

The foot and ankle specimens are naturally placed in the loading device, and $10 \mathrm{~N}$ force is applied to correctly fix the foot and ankle. Two metal screws are used to fix the subtalar joint from the anterior talus neck to the calcaneus. After fixation, CT scans were performed to confirm the screws position to ensure the subtalar joints were stable and fixed. Then, the tests were carried out the same as when the subtalar joint was intact, and the corresponding medial and lateral pressures of the ankle joint under different working conditions were recorded.

\section{Statistical analysis}

SPSS V.23 software (IBM Inc., New York) was used for the data analysis. Paired student t-test was employed to determine the significant differences for pressure differences. The level of significance was set to a p-value $<0.05$.

\section{Results}

1. When the tibia was varus

(1) The subtalar joint was intact

From 0 degree to 20 degrees varus, the pressure on the medial side of the ankle joint gradually decreases from $2340.4 \pm 646.7 \mathrm{kPa}$ to $1618.7 \pm 451.5 \mathrm{kPa}(\mathrm{p}<0.05)$. The pressure on the lateral side of the ankle joint decreases from $2083.8 \pm 487.2 \mathrm{kPa}$ (0 degree), gradually increased to $2280.7 \pm 376.1 \mathrm{kPa}$ (10 degrees), $(p<0.05)$, and then gradually decreased to $1862.9 \pm 342.6 \mathrm{kPa}$ (20 degrees). There was a turning point when the tibia was in 10 degrees varus on the lateral side of ankle.

(2) The subtalar joint was fused

The pressure on the medial ankle joint gradually decreased from $2457.4 \pm 427.5 \mathrm{kPa}$ to $2209.8 \pm 382.1 \mathrm{kPa}$ $(p<0.05)$. The pressure on the lateral side of the ankle joint gradually decreased from $2148.6 \pm 426.3 \mathrm{kPa}$ at 0 degrees to $1853.7 \pm 331.5 \mathrm{kPa}$ at 20 degrees $(\mathrm{p}<0.05)$.

2. When the tibia was valgus 
(1) The subtalar joint was intact

When tibial valgus angle gradually increased from 0 degrees to 20 degrees, the pressure on the inside of the ankle joint gradually increased from $2021.9 \pm 427.3 \mathrm{kPa}$ to $2517.7 \pm 457.2 \mathrm{kPa}(P<0.05)$. The pressure on the outside of the ankle joint gradually decreases from 0 degrees $2116.3 \pm 364.8 \mathrm{kPa}$ to $1893.9 \pm 321.5 \mathrm{kPa}$ $(P<0.05)$.

(2) The subtalar joint was fused

The pressure on the medial ankle joint gradually decreased from $2231.4 \pm 372.0 \mathrm{kPa}$ to $1982.5 \pm 381.7 \mathrm{kPa}$ $(P<0.05)$. The pressure on the outside of the ankle joint gradually increased from $2052.6 \pm 365.2 \mathrm{kPa}$ at 0 degrees to $2305.3 \pm 393.5 \mathrm{kPa}$ at 20 degrees. $(P<0.05)$ When the subtalar joint was fused, the change of ankle pressure was completely opposite to the ankle pressure when the subtalar joint was intact.

\section{Discussion}

Ankle arthritis is a debilitating disease usually with varus or valgus deformity. The alignment of ankle and hindfoot is crucial for realizing the success of surgeries. An important goal of operation of ankle arthritis is to achieve normal alignment. The subtalar joint plays a vital role in maintaining the normal position of talus to the tibia $[13,14]$. We must master the balancing mechanism of ankle and subtalar joint for a promising operative result.

To date, the etiology of primary ankle osteoarthritis is not well established. The compensatory mechanism is still controversial. Takakura thought that the ankle joint varus was compensated by subtalar valgus. If the ankle varus deformity exceeded the possible subtalar compensation, there was stress concentration on the medial part of ankle joint [15]. However, it was just a hypothesis and surgeon's experience. Hayashi et al. reviewed 133 ankles and found that the varus inclination of tibial articular surface progressed by stages, while the subtalar valgus inclination progressed on mild and intermediate stages [16]. For more severe stages, the subtalar surface converted to varus inclination. Wang et al. documented subtalar compensation for malaligned ankle, especially varus ankle, by evaluating weight-bearing radiographs of 233 ankles [17]. Burssens et al. reported that the subtalar joint's orientation was not associated with an opposite angulation relative to the hindfoot deformity. This suggested that not every hindfoot deformity was compensated by the subtalar joint [18]. Numerous studies investigated the subtalar compensation mechanism with clinical researches or radiographic studies. However, few studies demonstrated using basic or biomechanical research.

In this study, we firstly stimulated the varus and valgus tibia without subtalar fusion. Secondly, we fused the subtalar joint to evaluate the changes of ankle pressure with the varus and valgus tibia. We noticed different ankle pressure change comparing the intact and fused subtalar joint. When the subtalar joint is intact and the tibial varus angle gradually increases from 0 degrees to 20 degrees, the pressure on the lateral side of the ankle joint decreases from $2083.8 \pm 487.2 \mathrm{kPa}$ ( 0 degrees) gradually increased to $2280.7 \pm 376.1 \mathrm{kPa}$ (10 degrees), and then gradually decreases to $1862.9 \pm 342.6 \mathrm{kPa}$ (20 degrees). However, 
when the subtalar joint was fused, this phenomenon did not happen. We hypothesized that the subtalar joint has a compensatory mechanism. When the supramalleolar part turns finite varus deformity, the subtalar joint will turn eversion. With the supramalleolar varus progressing, the compensatory mechanism of subtalar will not work. However, when the tibia turned valgus, the pressure change of whether a medial or lateral aspect of ankle joint was opposite between the intact subtalar and fused subtalar groups. In the tibial valgus group, we did not observed the increasing and then decreasing process. We hypothesized that the inversion compensation of the subtalar joint is more likely to occur than the eversion compensation. Thus, for the correction of varus or valgus ankle arthritis, we should not only focus on the supramalleolar deformity, but also considered the subtalar joint.

There are some limitations of this study. First, the compensatory of ankle joint and hindfoot is complex. It relates to many factors, including ankle joint, subtalar joint, even talonavicular joint and ligaments surrounding talus. Does the ankle joint itself have its own compensatory mechanism? It is unknown. Secondly, it is a static biomechanical study, the tendons, muscles of ankle balancing in vivo. Further studies are needed to explore these questions.

\section{Conclusions}

For the varus ankle, the pressure on the lateral aspect of the ankle joint will increase due to the subtalar valgus inclination. When the varus deformity progressed, the lateral pressure decreased. When the subtalar joint is fused, this compensatory phenomenon does not happen. However, when the tibia turned valgus, the change of pressure of whether a medial or lateral aspect of ankle joint was opposite between the intact subtalar group and fused subtalar group. In the tibial valgus group, we did not observe the increasing and then decreasing process. Therefore, we hypothesized that the inversion compensation of the subtalar joint is more likely to occur than the eversion compensation.

\section{Declarations}

\section{Acknowledgements}

The authors gratefully acknowledge all collaborating researchers in collecting information during this study. We would also like to thank the subjects whose participation made this investigation possible.

\section{Authors' contributions}

$\mathrm{MH}$ : literature research, experimental studies, statistical analysis and manuscript preparation. XYX: experimental studies and manuscript editing. JM concepts and study design, experimental studies. All authors read and approved the final manuscript.

\section{Funding}


This research did not receive any specific grant from funding agencies in the public, commercial, or notfor-profit sectors.

\section{Availability of data and materials}

All relevant data are available from the corresponding author.

Ethics approval and consent to participate

Our research was approved by the ethics committee of School of Medicine, Tongji University. We also followed the Declaration of Helsinki and relevant policies in China. The cadaveric specimens used in this study were provided by the Collage of Medicine, Shanghai Jiaotong University. Written informed consent to participate in this study was obtained from the legal representative of each patient.

Consent for publication

Not Applicable.

Competing interests

The authors declare that they have no competing interests.

\section{References}

1. Barg A, Pagenstert GI, Hugle T, Gloyer M, Wiewiorski M, Henninger HB, Valderrabano V: Ankle osteoarthritis: etiology, diagnostics, and classification. Foot Ankle Clin 2013, 18(3):411-426.

2. Hintermann B, Knupp M, Barg A: Supramalleolar Osteotomies for the Treatment of Ankle Arthritis. J Am Acad Orthop Surg 2016, 24(7):424-432.

3. Saltzman CL, Salamon ML, Blanchard GM, Huff T, Hayes A, Buckwalter JA, Amendola A: Epidemiology of ankle arthritis: report of a consecutive series of 639 patients from a tertiary orthopaedic center. lowa Orthop J 2005, 25:44-46.

4. Valderrabano V, Horisberger M, Russell I, Dougall H, Hintermann B: Etiology of ankle osteoarthritis. Clin Orthop Relat Res 2009, 467(7):1800-1806.

5. Horisberger M, Valderrabano V, Hintermann B: Posttraumatic ankle osteoarthritis after ankle-related fractures. J Orthop Trauma 2009, 23(1):60-67.

6. Hintermann B, Knupp M, Barg A: Peritalar instability. Foot Ankle Int 2012, 33(5):450-454.

7. Hintermann B, Knupp M, Barg A: Joint-preserving surgery of asymmetric ankle osteoarthritis with peritalar instability. Foot Ankle Clin 2013, 18(3):503-516. 
8. Zhu Y, Li X, Xu X: Ankle joint pressure change in varus malalignment of the tibia. BMC Musculoskelet Disord 2020, 21(1):148.

9. Bendall S, Halliwell P, Goldberg A, Robinson A: Ankle Arthritis Networking: Getting the right treatment to the right patient first time. Foot Ankle Surg 2021.

10. Xu Y, Zhu Y, Xu XY: Ankle joint distraction arthroplasty for severe ankle arthritis. BMC Musculoskelet Disord 2017, 18(1):96.

11. Walter RP, Walker RW, Butler M, Parsons S: Arthroscopic subtalar arthrodesis through the sinus tarsi portal approach: A series of 77 cases. Foot Ankle Surg 2018, 24(5):417-422.

12. Klerken T, Kosse NM, Aarts CAM, Louwerens JWK: Long-term results after triple arthrodesis: Influence of alignment on ankle osteoarthritis and clinical outcome. Foot Ankle Surg 2019, 25(2):247-250.

13. Mullaji A, Shetty GM: Persistent hindfoot valgus causes lateral deviation of weightbearing axis after total knee arthroplasty. Clin Orthop Relat Res 2011, 469(4):1154-1160.

14. Norton AA, Callaghan JJ, Amendola A, Phisitkul P, Wongsak S, Liu SS, Fruehling-Wall C: Correlation of knee and hindfoot deformities in advanced knee OA: compensatory hindfoot alignment and where it occurs. Clin Orthop Relat Res 2015, 473(1):166-174.

15. Takakura Y, Tanaka Y, Kumai T, Tamai S: Low tibial osteotomy for osteoarthritis of the ankle. Results of a new operation in 18 patients. J Bone Joint Surg Br 1995, 77(1):50-54.

16. Hayashi K, Tanaka Y, Kumai T, Sugimoto K, Takakura Y: Correlation of compensatory alignment of the subtalar joint to the progression of primary osteoarthritis of the ankle. Foot Ankle Int 2008, 29(4):400-406.

17. Wang B, Saltzman CL, Chalayon O, Barg A: Does the subtalar joint compensate for ankle malalignment in end-stage ankle arthritis? Clin Orthop Relat Res 2015, 473(1):318-325.

18. Burssens ABM, Buedts K, Barg A, Vluggen E, Demey P, Saltzman CL, Victor JMK: Is Lower-limb Alignment Associated with Hindfoot Deformity in the Coronal Plane? A Weightbearing CT Analysis. Clin Orthop Relat Res 2020, 478(1):154-168.

\section{Figures}




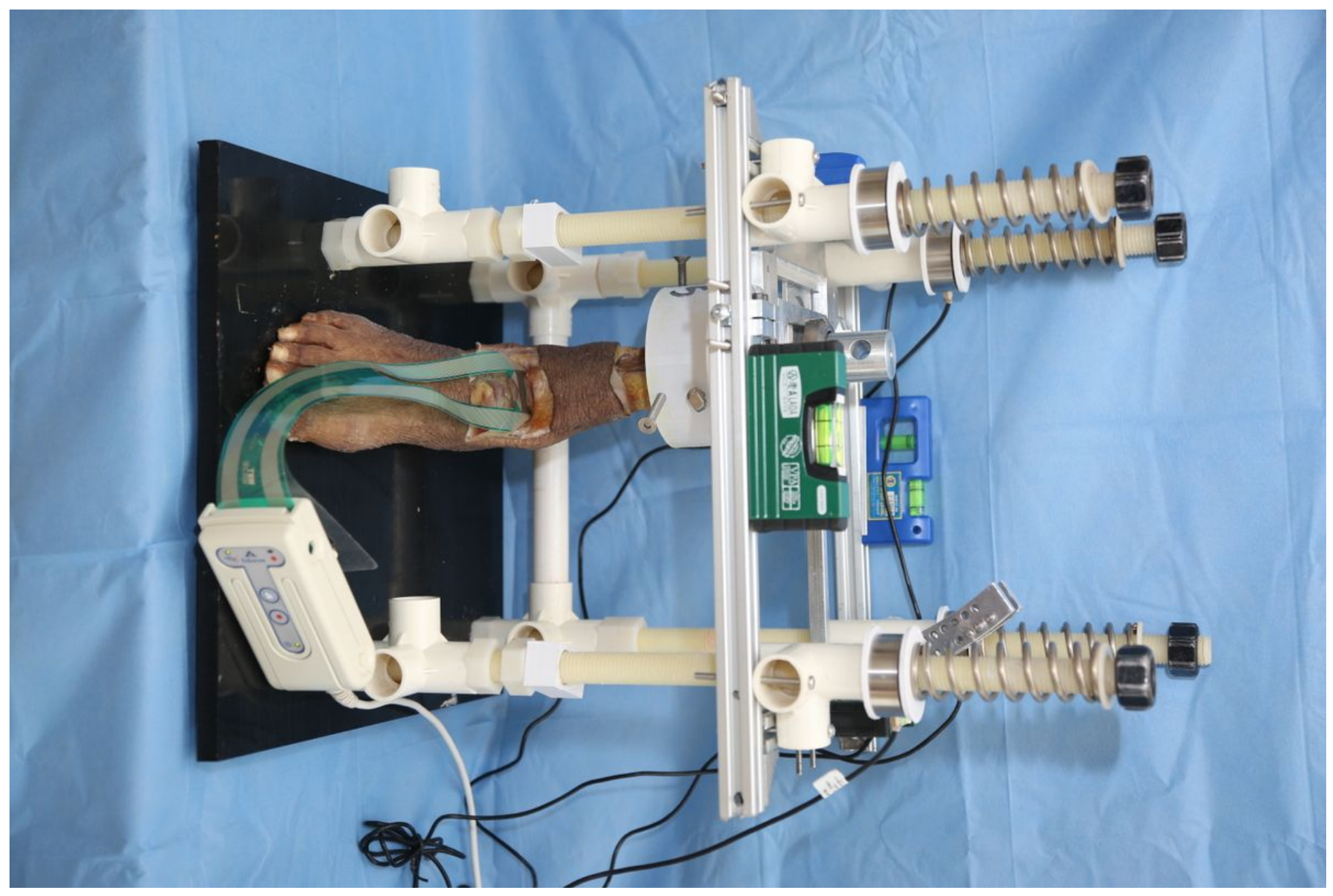

Figure 1

The specimen was fixed into the shell using dental gypsum. 


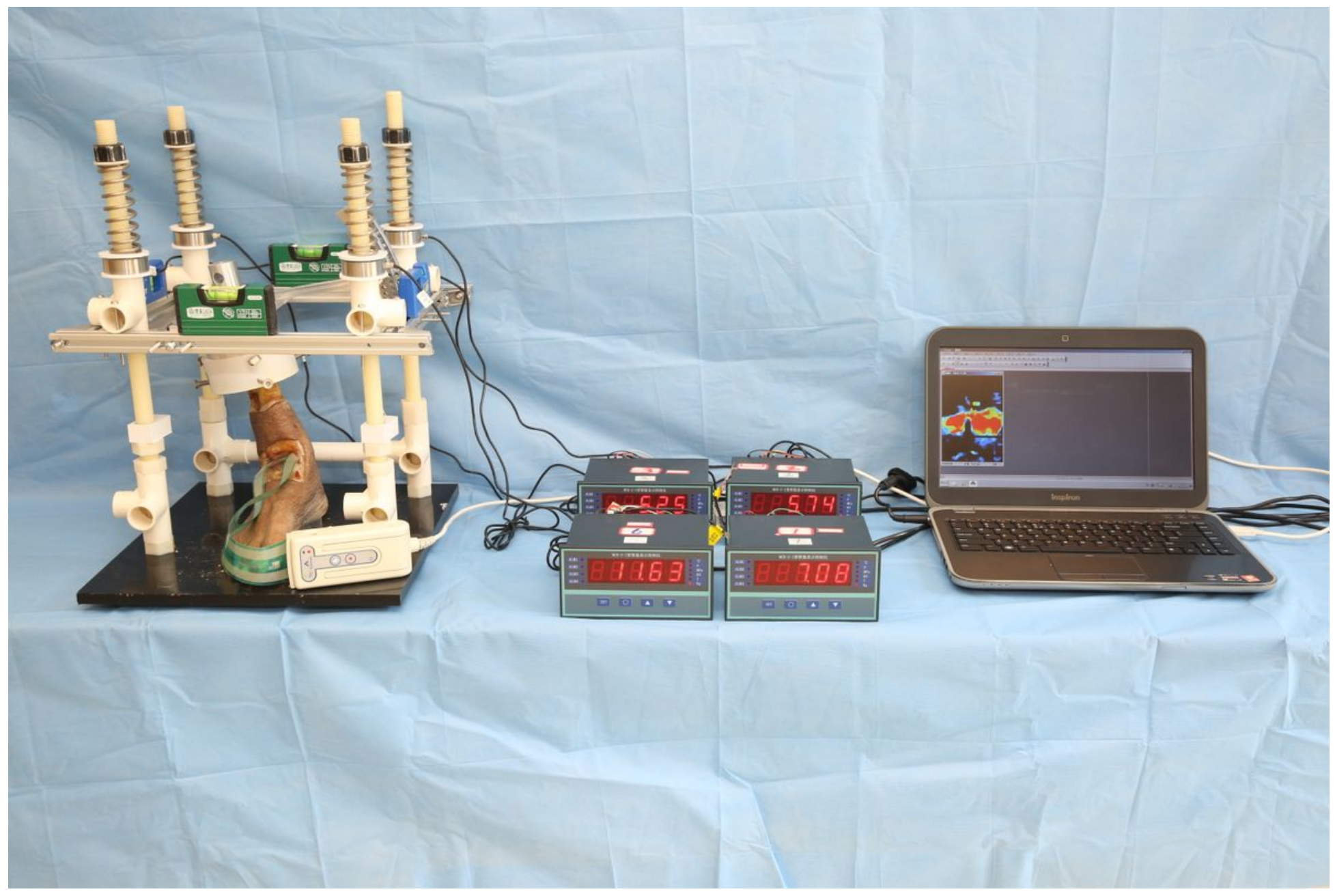

Figure 2

The specimen was connected to force sensor and monitor. 


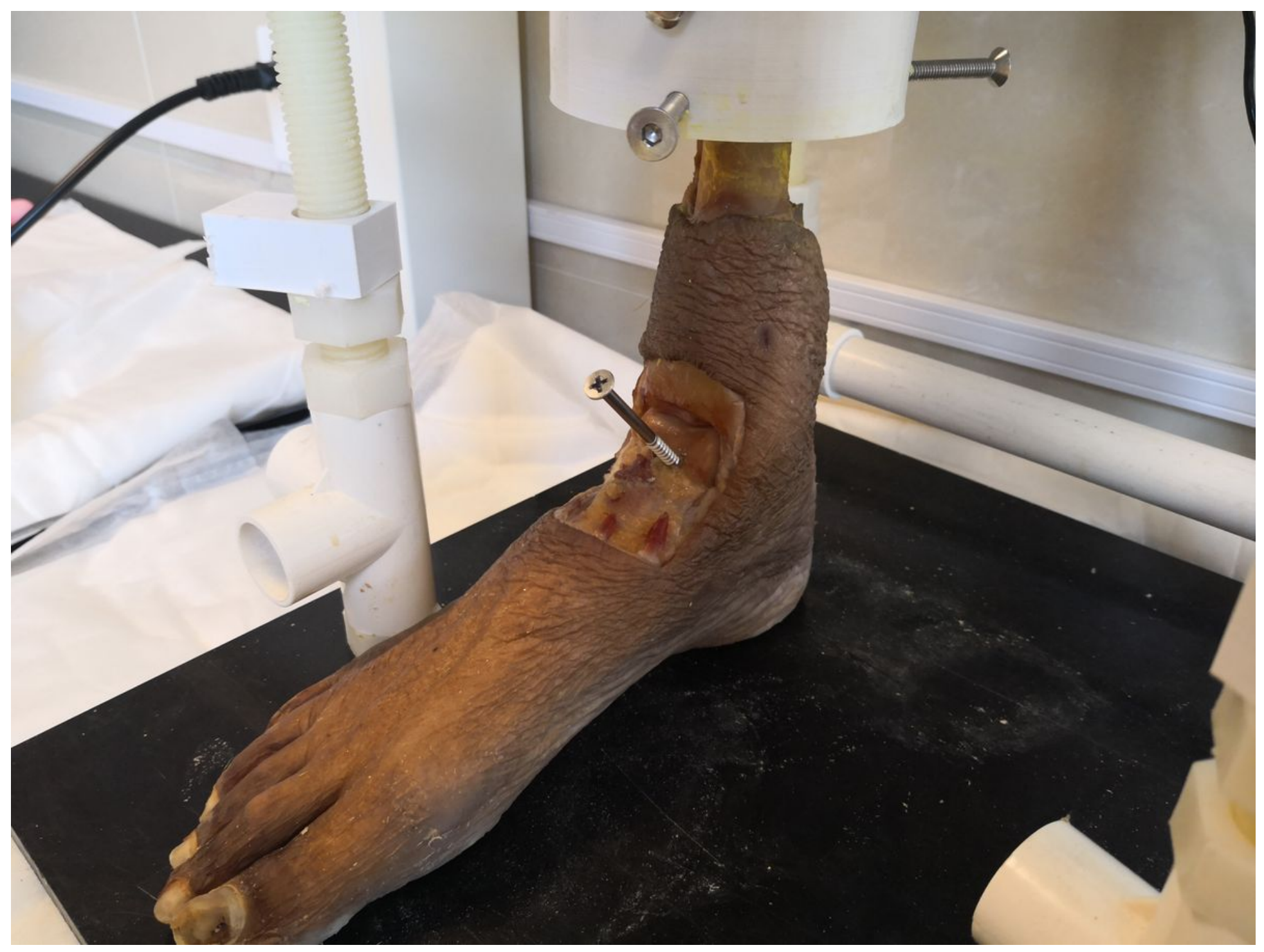

Figure 3

The subtalar joint was fused using two metal screws. 


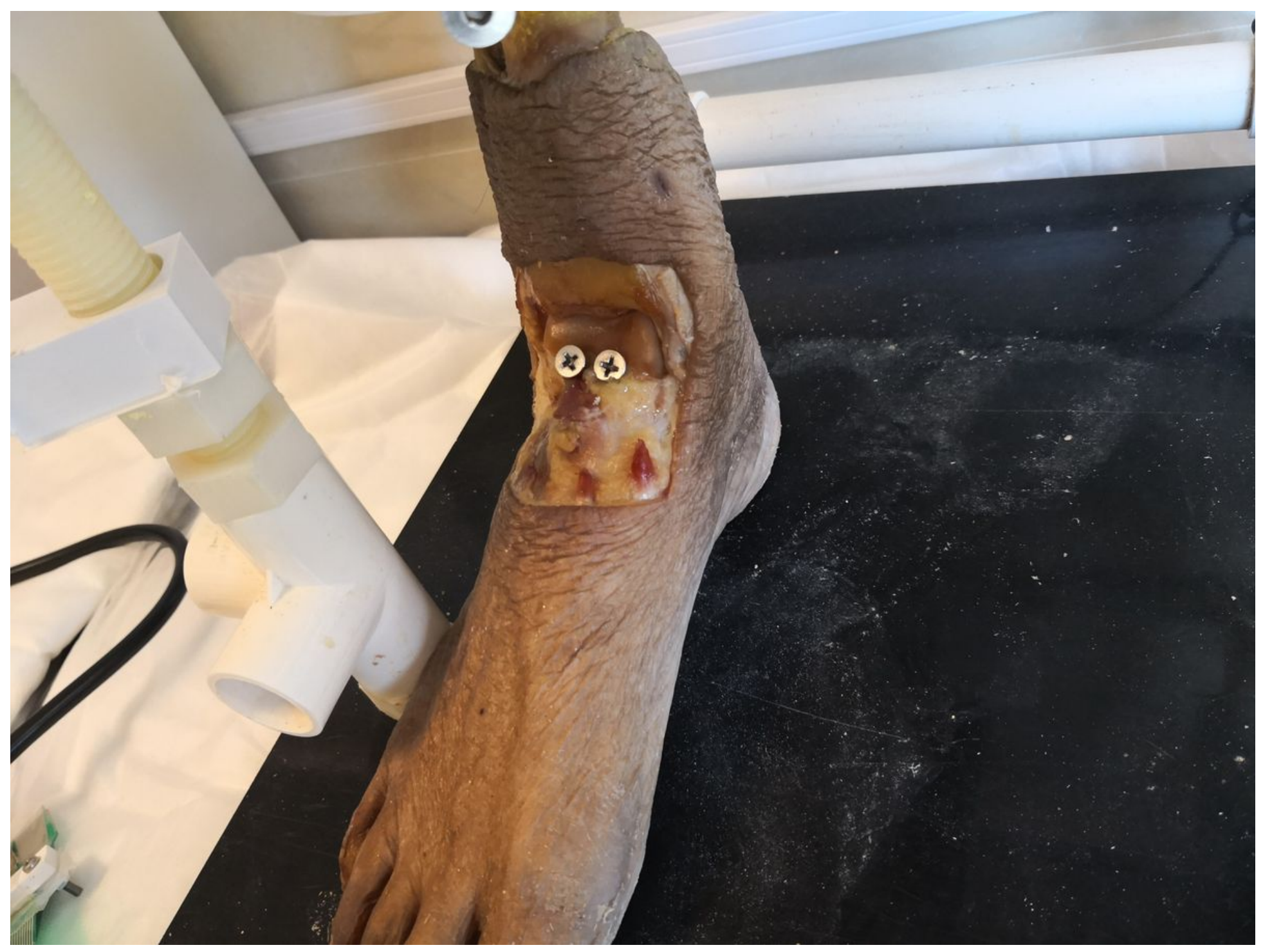

Figure 4

The anterior view of specimen, fused by two metal screws.

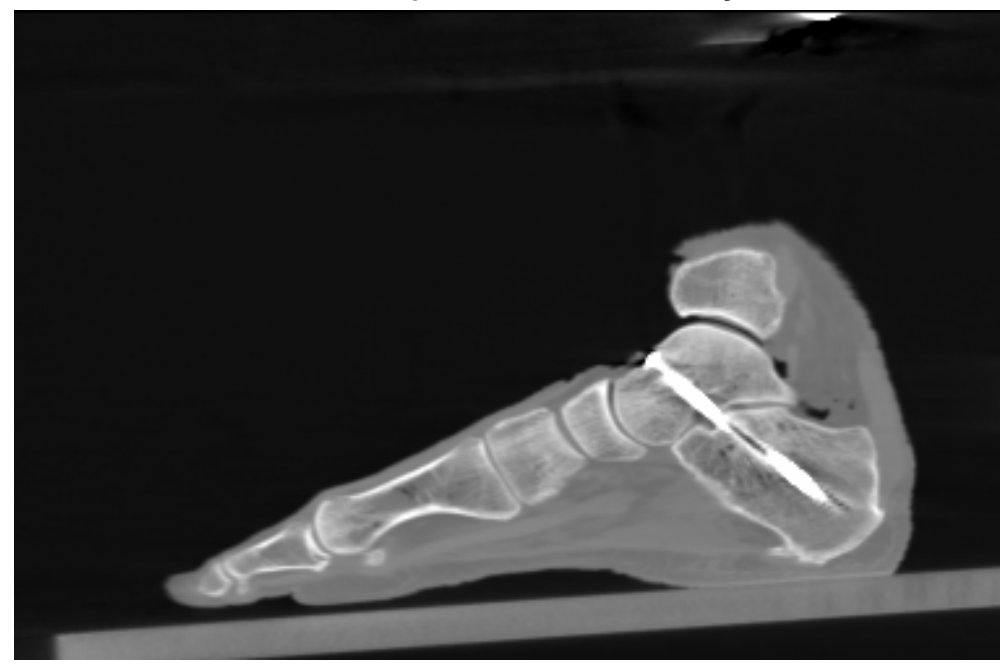

Figure 5 
This CT scan showed the fusion of subtalar joint.

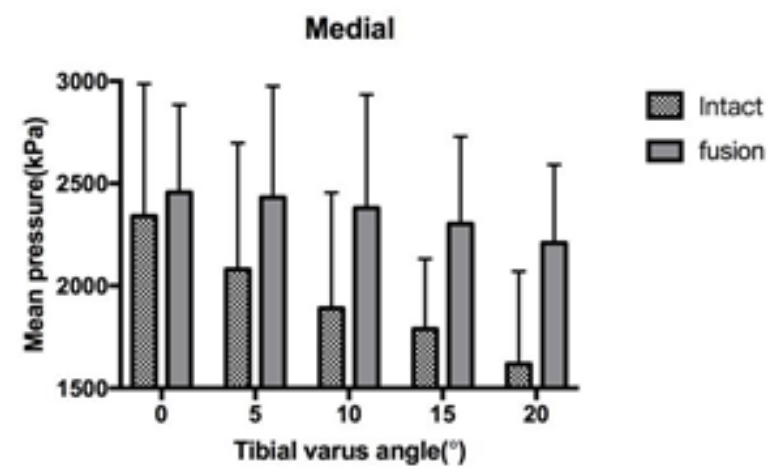

Lateral

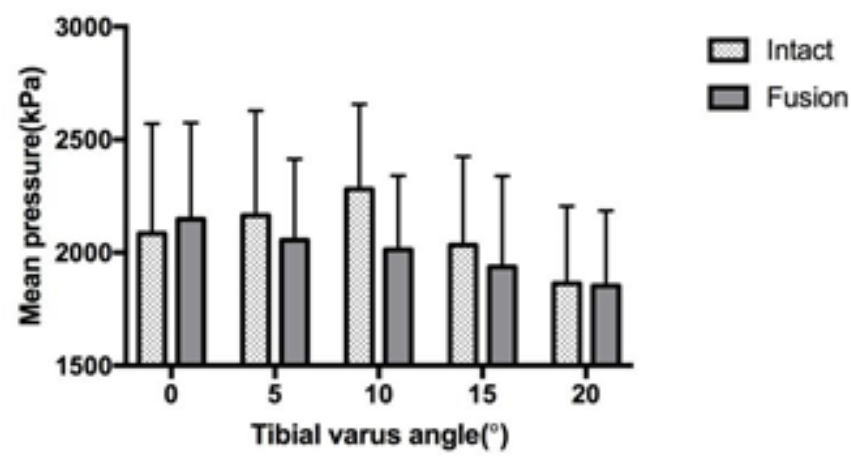

\section{Figure 6}

A: The pressure of medial aspect of ankle joint decreased as tibial varus progressed. B: The pressure of lateral aspect of ankle joint changed as the tibial varus progressed.

Medial

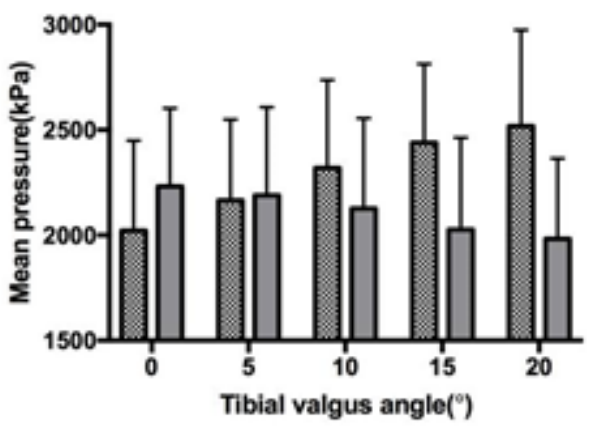

Lateral

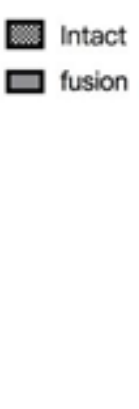

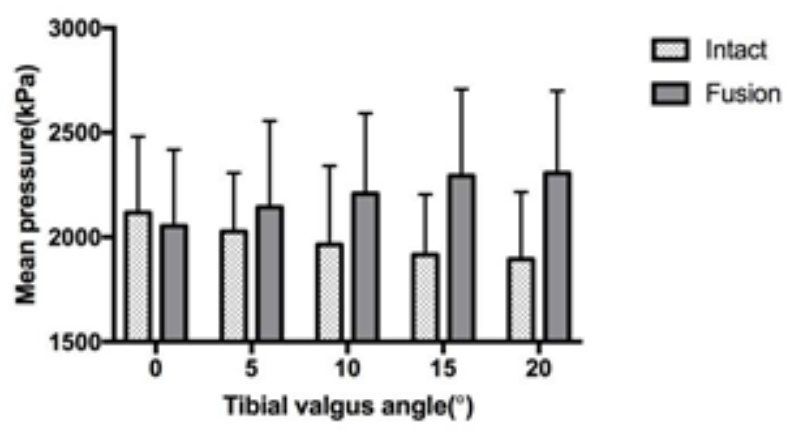

Figure 7

A: The pressure of medial aspect of ankle joint changed as tibial valgus progressed. B: The pressure of lateral aspect of ankle joint changed as the tibial varus progressed.
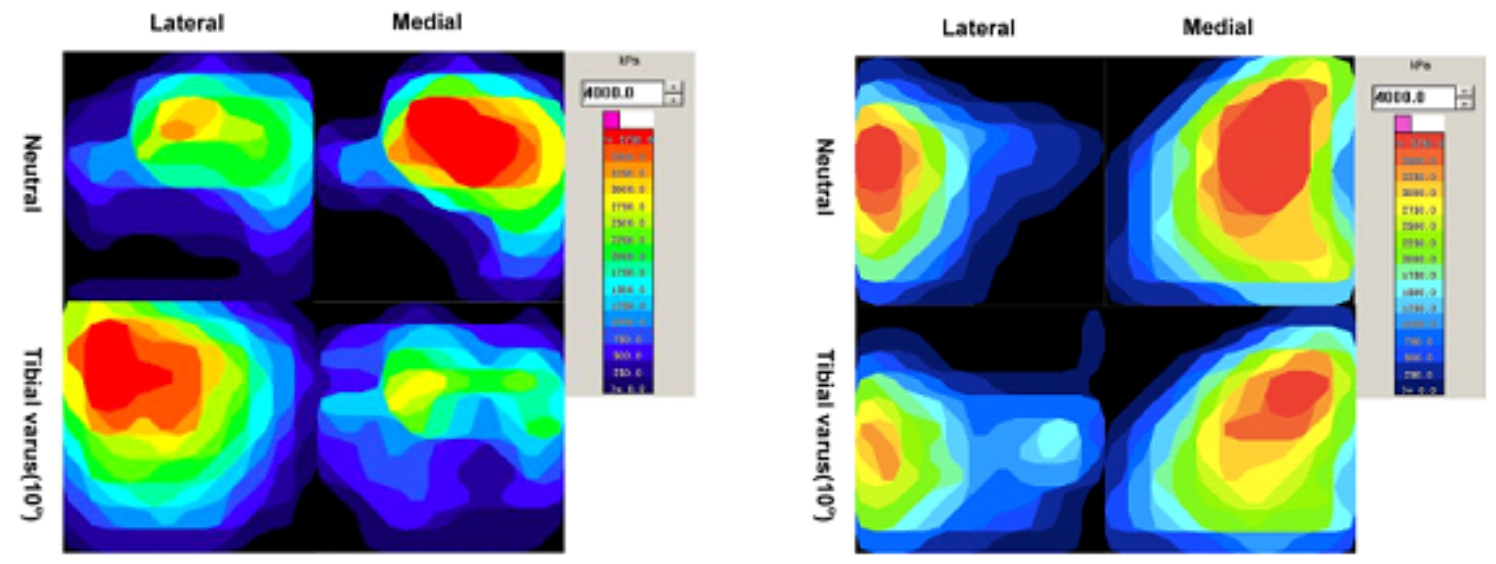

Figure 8 
A: The subtalar joint was intact and the tibial varus angle was $10^{\circ}$, noting the stress concentration on the lateral side of the ankle joint. B: The subtalar joint was fused and the tibial varus angle was $10^{\circ}$, no stress concentration was observed on the lateral side of the ankle joint. 